To appear in Integral Transforms and Special Functions

Vol. 00, No. 00, Month 201X, 1-16

\title{
On Laguerre-Hahn affine Orthogonal Polynomials on the Unit Circle from matrix Sylvester equations
}

\author{
M.N. Rebocho ${ }^{a}$ * \\ ${ }^{a}$ Departmento de Matemática, Universidade da Beira Interior, 6201-001 Covilhã, Portugal; \\ CMUC, Apartado 3008, EC Santa Cruz, 3001-501 Coimbra, Portugal \\ (Received 00 Month 201X; final version received 00 Month 201X)
}

\begin{abstract}
In this paper are derived recurrences for the reflection coefficients of Laguerre-Hahn affine orthogonal polynomials on the unit circle, including a form of the discrete Painlevé equations $d P_{V}$. The technique is based on the knowledge of the first order differential equation for the Carathéodory function, combined with a re-interpretation, in the formalism of matrix Sylvester equations, of compatibility conditions for the differential systems satisfied by the polynomials.
\end{abstract}

Keywords: Hermitian linear functionals; OPUC; Laguerre-Hahn affine class; Sylvester equations; discrete Painlevé equations.

AMS Subject Classification: 33C45; 33C47; 33D45.

\section{Motivation}

Laguerre-Hahn affine orthogonal polynomials on the unit circle are related to Carathéodory functions, $F$, that satisfy first order differential equations with polynomial coefficients $[1,2]$

$$
z A F^{\prime}=C F+D .
$$

Well-known families of such polynomials include the semi-classical orthogonal polynomials on the unit circle - characterized through a rational logarithmic derivative for the weight function, equivalently, through an ODE (1) with a specific polynomial $D[1-3]$ - as well as some of their perturbations, for instance, the ones studied in [4-6].

The analysis of relations between differential properties of sequences of orthogonal polynomials and differential properties of the corresponding Carathéodory function through ODEs (1) is an often encountered problem in the literature of Orthogonal Polynomials. A central aim is to deduce properties of the polynomials and their recurrence coefficients from the knowledge of a differential equation satisfied by the weight function or from (1). Such a problem has been studied in a vast list of works, e.g., in [7-11] as well as in many of the references therein, where one emphasises the semi-classical character of the orthogonal polynomials (when orthogonality on the real line, the Carathéodory function is replaced by the Stieltjes function). Connections with integrable systems, including

\footnotetext{
* Email: mneves@ubi.pt
} 
continuous and discrete Painlevé equations, are well-known (see [7, 8, 10, 12-15]). For a comprehensive overview of Painlevé equations, see [16, 17].

The study of differential systems for sequences of Laguerre-Hahn affine orthogonal polynomials on the unit circle is known at least since [2], and it has been recently revisited in $[18,19]$. In general and basic terms (details are given in Section 3), having the differential system for the sequence of polynomials related to (1), say $\left\{\phi_{n}\right\}_{n \geq 0}$, by a standard method based on the recurrence relations, one obtains the so-called compatibility conditions, written in the matrix form as

$$
\mathcal{B}_{n} \mathcal{A}_{n}-\mathcal{A}_{n} \mathcal{B}_{n-1}=\mathcal{C}_{n}
$$

where $\mathcal{A}_{n}$ are the transfer matrices (cf. Section 2) and $\mathcal{B}_{n}, \mathcal{C}_{n}$ are matrices having polynomial entries containing the information on the coefficients of (1).

In this work the above equations (2) are re-analysed within the theory of matrix Sylvester equations (cf. Sections 3 and 4). In Proposition 3.3 is given a closed form solution of (2), which is equivalent to the two scalar equations stated in Corollary 3.4,

$$
\begin{aligned}
& a_{n}\left(l_{n, 1}+l_{n-1,1}-(n-1) A\right)=\Theta_{n, 1}-z \Theta_{n-1,1}, \quad n \geq 1, \\
& l_{n, 1}-l_{n-1,1}=A-a_{n} \Theta_{n, 2}+z \bar{a}_{n} \Theta_{n-1,1}, \quad n \geq 1,
\end{aligned}
$$

where $l_{n, 1}, \Theta_{n, j}, j=1,2$, are polynomials, $A$ is the same as in (1), and $a_{n}$ are the reflection coefficients of $\left\{\phi_{n}\right\}_{n \geq 0}$ (cf. Section 2). Eqs. (3)-(4) constitute one of the tools for providing recurrences for the reflection coefficients of orthogonal polynomials related to (1). Applications of these results are shown for the so-called generalized Jacobi polynomials on the unit circle [20, Section 4], as well as for some of its perturbations falling in the Laguerre-Hahn affine class (non semi-classical): several recurrences are derived, including a form of the discrete Painlevé equation $\mathrm{dP}_{V}$ (cf. Section 4).

The remainder of the paper is organized as follows. In Section 2 is given the basic results and notations to be used in the forthcoming sections. In Section 3 is deduced the closed form solution of the Sylvester matrix equations contained in Theorem 3.1, leading to the above Eqs. (3)-(4). Section 4 is devoted to the derivation of recurrences for the generalized Jacobi polynomials and its perturbations.

\section{Preliminary results and notations}

Let $\mathbb{T}=\{z \in \mathbb{C}:|z|=1\}$ be the unit circle, let $\Lambda=\operatorname{span}\left\{z^{k}: k \in \mathbb{Z}\right\}$ be the linear space of Laurent polynomials with complex coefficients, and $\mathbb{P}=\operatorname{span}\left\{z^{k}: k \in \mathbb{N}_{0}\right\}$ its linear subspace of polynomials. We consider a Hermitian linear functional $u: \Lambda \rightarrow \mathbb{C}$, that is, its sequence of moments $u_{n}=\left\langle u, z^{-n}\right\rangle, n \in \mathbb{Z}$, is such that $u_{-n}=\bar{u}_{n}, \forall n \geq 0$. With $u$ we associate the Toeplitz matrices

$$
\mathbf{T}_{n}=\left[\begin{array}{ccc}
u_{0} & \cdots & \bar{u}_{n} \\
\vdots & \ddots & \vdots \\
u_{n} & \cdots & u_{0}
\end{array}\right], \quad n \geq 0
$$

and we set $\Delta_{n}=\operatorname{det}\left(\mathbf{T}_{n}\right), n \geq 0$. By convention, $\Delta_{-1}=1$.

Assuming $\Delta_{n} \neq 0, \forall n \geq 0$, that is, $u$ is regular, one defines the sequence of orthogonal polynomials with respect to $u,\left\{\phi_{n}\right\}_{n \geq 0}$, such that $\operatorname{deg}\left(\phi_{n}\right)=n$, and

$$
\left\langle u, \phi_{n}(z) z^{-k}\right\rangle=h_{n} \delta_{n, k}, \quad 0 \leq k \leq n, \quad h_{n} \neq 0, \quad n \geq 0 .
$$


In the sequel we will take each $\phi_{n}$ as monic, i.e., $\phi_{n}(z)=z^{n}+$ lower degree terms, and $\left\{\phi_{n}\right\}_{n \geq 0}$ will be called a monic orthogonal polynomial sequence sequence (MOPS). Note that the MOPS can be given as [21]

$$
\phi_{n}(z)=\frac{1}{\Delta_{n-1}}\left|\begin{array}{cccc}
u_{0} & \bar{u}_{1} & \cdots & \bar{u}_{n} \\
\vdots & \vdots & & \vdots \\
u_{n-1} & u_{n-2} & \cdots & \bar{u}_{1} \\
1 & z & \cdots & z^{n}
\end{array}\right|, \quad n \geq 1, \quad \phi_{0}(z)=1
$$

Additionally, if $\Delta_{n}>0, \forall n \geq 0$, that is, $u$ is positive definite, then $u$ has an integral representation given in terms of a finite and positive Borel measure, $\mu$, with infinite support on $\mathbb{T}$, such that

$$
\langle u, f(z)\rangle=\frac{1}{2 \pi} \int_{0}^{2 \pi} f\left(e^{i \theta}\right) d \mu(\theta), \quad f \in \Lambda
$$

In such a case, the $\phi_{n}$ 's are the so-called orthogonal polynomials on the unit circle (OPUC) [22]. If $\mu$ is absolutely continuous with respect to the Lebesgue measure on $\mathbb{T}$, defined in terms of a weight, say $w$, then we will also say that $\left\{\phi_{n}\right\}_{n \geq 0}$ is a sequence of orthogonal polynomials with respect to $w$.

For future purposes we introduce the operator $*_{p}$, where $p$ is a positive integer, as follows. Given an analytic function $f, f^{*_{p}}(z)=z^{p} \bar{f}(1 / z)$, that is, if $f(z)=\sum_{k=0}^{+\infty} b_{k} z^{k}$, then $f^{*_{p}}(z)=\sum_{k=0}^{+\infty} \bar{b}_{k} z^{-k+p}$. Whenever $f$ is a polynomial of degree $p$, the index $p$ will be omitted and it will be written $f^{*}$ instead of $f^{*_{p}}$. In such a case, $f^{*}$ is called the reversed polynomial of $f$.

MOPS on the unit circle satisfy the Szegö recurrence relations [21], for all $n \geq 1$ :

$$
\begin{aligned}
& \phi_{n}(z)=z \phi_{n-1}(z)+a_{n} \phi_{n-1}^{*}(z), \\
& \phi_{n}^{*}(z)=\phi_{n-1}^{*}(z)+\bar{a}_{n} z \phi_{n-1}(z) .
\end{aligned}
$$

Define the vectors $\Psi_{n}(z)=\left[\begin{array}{l}\phi_{n}(z) \\ \phi_{n}^{*}(z)\end{array}\right], n \geq 0$. The Szegö recurrence relations can be written as

$$
\Psi_{n}(z)=\mathcal{A}_{n} \Psi_{n-1}(z), \quad \mathcal{A}_{n}=\left[\begin{array}{cc}
z & a_{n} \\
\bar{a}_{n} z & 1
\end{array}\right], \quad n \geq 1
$$

The matrices $\mathcal{A}_{n}$, called transfer matrices, play an important role in the theory of orthogonal polynomials on the unit circle. A comprehensive study, related to the theory of matrix valued functions, can be found in [23]. The complex numbers $a_{n}$ in (7)-(9), called reflection coefficients, satisfy $\left|a_{n}\right| \neq 1, \forall n \geq 1$ (in the regular case) or $\left|a_{n}\right|<1, \forall n \geq 1$ (in the positive definite case). The converse result, commonly referred to as Favard's Theorem on the unit circle, also holds, that is, any sequence of monic polynomials $\left\{\phi_{n}\right\}_{n>0}$ satisfying one of the difference relations (7)-(9) with $\left|a_{n}\right| \neq 1, \forall n \geq 1$, (respectively $\left|a_{n}\right|<1, \forall n \geq 1$ ) constitutes a sequence of orthogonal polynomials with respect to a Hermitian and regular linear functional (respectively positive definite Hermitian linear functional) [23, 24].

Some central functions of the present work are defined as follows (see [25, 26]). Given a MOPS $\left\{\phi_{n}\right\}_{n \geq 0}$ with respect to $u$, one defines the sequence of associated polynomials 
of the second kind, $\left\{\Omega_{n}\right\}_{n \geq 0}$, as

$$
\Omega_{n}(z)=\left\langle u_{\theta}, \frac{e^{i \theta}+z}{e^{i \theta}-z}\left(\phi_{n}\left(e^{i \theta}\right)-\phi_{n}(z)\right)\right\rangle, \quad n \geq 1, \quad \Omega_{0}(z)=1 .
$$

One defines the generating function of the moments of $u$, also called formal Carathéodory function, having expansions

$$
F(z)=u_{0}+2 \sum_{k=1}^{+\infty} u_{k} z^{k},|z|<1, \quad F(z)=-u_{0}-2 \sum_{k=1}^{+\infty} u_{-k} z^{-k},|z|>1
$$

In the positive definite case, whenever $u$ has an integral representation as in $(6), F$ is the Carathéodory function of the measure $\mu$,

$$
F(z)=\frac{1}{2 \pi} \int_{0}^{2 \pi} \frac{e^{i \theta}+z}{e^{i \theta}-z} d \mu(\theta)
$$

The sequence of functions of the second kind, $\left\{Q_{n}\right\}_{n \geq 0}$, is defined as

$$
Q_{n}(z)=\Omega_{n}(z)+F(z) \phi_{n}(z), \quad n \geq 1, \quad Q_{0}(z)=F(z),
$$

and the functions $Q_{n}^{*_{n}}$, which will be denoted by $Q_{n}^{*}$, are defined as

$$
Q_{n}^{*}(z)=\Omega_{n}^{*}(z)-F(z) \phi_{n}^{*}(z), \quad n \geq 1, \quad Q_{0}^{*}(z)=-F(z) .
$$

Note that $\left\{Q_{n}\right\}_{n \geq 0}$ satisfies the recurrence relation, for all $n \geq 1$,

$$
\begin{aligned}
& Q_{n}(z)=z Q_{n-1}(z)-a_{n} Q_{n-1}^{*}(z), \\
& Q_{n}^{*}(z)=Q_{n-1}^{*}(z)-\bar{a}_{n} z Q_{n-1}(z) .
\end{aligned}
$$

Set

$$
\phi_{n}(z)=z^{n}+\tau_{n} z^{n-1}+\cdots+k_{n} z+a_{n}, \quad n \geq 0 .
$$

Useful identities, to be used in the sequel, are listed below:

$$
\begin{aligned}
& \tau_{n+1}=\tau_{n}+\bar{a}_{n} a_{n+1}, \quad n \geq 0, \quad \tau_{0}=0, \\
& k_{n+1}=a_{n}+a_{n+1} \bar{\tau}_{n}, \quad n \geq 0, \quad k_{0}=0,
\end{aligned}
$$

(following from (7)-(8)), as well as the asymptotic expansions, for all $n \geq 1$,

$$
\begin{aligned}
& Q_{n}(z)=2 h_{n} z^{n}-2 \bar{\tau}_{n+1} h_{n} z^{n+1}+\mathcal{O}\left(z^{n+2}\right), \quad|z|<1 \\
& Q_{n}(z)=2 a_{n+1} h_{n} z^{-1}+2\left(a_{n+2} h_{n+1}-a_{n+1} \tau_{n+1} h_{n}\right) z^{-2}+\mathcal{O}\left(z^{-3}\right), \quad|z|>1
\end{aligned}
$$

(following from (10)-(11)).

Finally, we define the Laguerre-Hahn affine class on the unit circle. Such a class is constituted by the sequences of orthogonal polynomials whose Carathéodory function satisfies a first order differential equation $[1,2]$

$$
z A F^{\prime}=C F+D, A \not \equiv 0, \quad A, C, D \in \mathbb{P} .
$$


The Laguerre-Hahn affine class includes the semi-classical class on the unit circle. The latter one is characterized through an ODE (17) with a specific polynomial $D$ (see $[3$, Corollary 5] and [1, 2]), or, equivalently, whenever $F$ is related to some weight $w$, through the rational logarithmic derivative for $w, \frac{w^{\prime}}{w}=\frac{C}{z A}$.

A more detailed study of the Laguerre-Hahn affine class can be found in [1], focusing on both, the approach through differential equations (17), and the approach through distributional equations for the corresponding linear functional. The interested reader is also referred to [3].

\section{Laguerre-Hahn affine identities from matrix Sylvester equations}

From Theorem 3 and Corollary 3 in [19] (where Eq. (21) below is now written without the typo) the following result holds.

Theorem 3.1. Let $F$ be a Carathéodory function satisfying a differential equation with polynomial coefficients $z A F^{\prime}=C F+D$, let $\left\{\phi_{n}\right\}_{n \geq 0}$ be the MOPS related to $F$. The transfer matrix of $\left\{\phi_{n}\right\}_{n \geq 0}, \mathcal{A}_{n}=\left[\begin{array}{cc}z & a_{n} \\ \bar{a}_{n} z & 1\end{array}\right]$, satisfies the matrix Sylvester equation

$$
\mathcal{B}_{n} \mathcal{A}_{n}-\mathcal{A}_{n} \mathcal{B}_{n-1}=\mathcal{C}_{n}, \quad \mathcal{C}_{n}=\left[\begin{array}{cc}
z A & 0 \\
\bar{a}_{n} z A & 0
\end{array}\right], \quad n \geq 1
$$

where $\mathcal{B}_{n}$ are matrices whose entries are bounded degree polynomials,

$$
\mathcal{B}_{n}=\left[\begin{array}{cc}
l_{n, 1} & -\Theta_{n, 1} \\
-\Theta_{n, 2} & l_{n, 2}
\end{array}\right]
$$

such that

$$
\begin{aligned}
& \operatorname{tr}\left(\mathcal{B}_{n}\right)=n A, \quad n \geq 1 \\
& \operatorname{det}\left(\mathcal{B}_{n}\right)=\operatorname{det}\left(\mathcal{B}_{1}\right)+A \sum_{k=1}^{n-1} l_{k, 2}, \quad n \geq 2,
\end{aligned}
$$

with $\operatorname{tr}\left(\mathcal{B}_{n}\right)$ and $\operatorname{det}\left(\mathcal{B}_{n}\right)$ denoting the trace and the determinant of $\mathcal{B}_{n}$, respectively, and

$$
\operatorname{det}\left(\mathcal{B}_{1}\right)=A\left(2 \bar{a}_{1} z A-h_{1} D+\left(\left|a_{1}\right|^{2}+1\right) C\right) /\left(2 h_{1}\right)-C^{2} / 4 .
$$

Remark 3.2. The detailed proof of Theorem 3.1 can be obtained from [19, Theorem 3, Corollary 3], by putting $B \equiv 0$ in Eqs. (9), (14) and (15) therein. Hence, the polynomials $l_{n, j}, \Theta_{n, j}, j=1,2$ in (19) are defined through the differential system

$$
\begin{aligned}
& z A \phi_{n}^{\prime}=\left(l_{n, 1}-C / 2\right) \phi_{n}-\Theta_{n, 1} \phi_{n}^{*}, \\
& z A\left(\phi_{n}^{*}\right)^{\prime}=\left(l_{n, 2}-C / 2\right) \phi_{n}^{*}-\Theta_{n, 2} \phi_{n},
\end{aligned}
$$

where $\Theta_{n, j}, j=1,2$, are given by $\Theta_{n, 1}=\tilde{\Theta}_{n} /\left(2 h_{n} z^{n}\right), \Theta_{n, 2}=\hat{\Theta}_{n} /\left(2 h_{n} z^{n}\right)$, with

$$
\tilde{\Theta}_{n}=\left\{z A\left(\frac{Q_{n}}{\phi_{n}}\right)^{\prime}-C \frac{Q_{n}}{\phi_{n}}\right\} \phi_{n}^{2}, \hat{\Theta}_{n}=\left\{z A\left(\frac{Q_{n}^{*}}{\phi_{n}^{*}}\right)^{\prime}-C \frac{Q_{n}^{*}}{\phi_{n}^{*}}\right\}\left(\phi_{n}^{*}\right)^{2} .
$$


The leading and trailing expansions of the $\Theta_{n}$ 's can be obtained through the expansions (15) and (16). The $l_{n}$ 's can be easily determined by equating coefficients in (23)-(24) once the $\Theta_{n}$ 's are known.

Furthermore, let us remark that Eq. (18) follows from the compatibility between the differential system (23)-(24) and the recurrence relations (9).

The previous theorem gives us the transfer matrix $\mathcal{A}_{n}$ as a solution of a matrix Sylvester equation

$$
\mathcal{B}_{n} X-X \mathcal{B}_{n-1}=\mathcal{C}_{n}
$$

According to the so-called Sylvester Theorem (see [27]), for each matrix $\mathcal{C}_{n}$, the matrix Sylvester equation (26) has a unique solution if, and only if, the spectrum of the matrices $\mathcal{B}_{n}$ and $\mathcal{B}_{n-1}$ are disjoint sets. The lemma that follows provides a closed form solution of (26).

Proposition 3.3. Let the matrix Sylvester equation (26) hold, with $\mathcal{B}_{n}$ given in (19). If, for each $z \in \mathbb{C}, \mathcal{B}_{n}(z)$ and $\mathcal{B}_{n-1}(z)$ have no common eigenvalue, then (26) is equivalent to

$$
\left(\mathcal{B}_{n}-l_{n-1,2} I\right) X=-(n-1) \mathcal{C}_{n}+\mathcal{B}_{n} z \mathcal{D}_{n}+z \mathcal{D}_{n} \mathcal{B}_{n-1}
$$

where $I$ denotes the identity matrix and $\mathcal{D}_{n}=\left[\begin{array}{cc}1 & 0 \\ \bar{a}_{n} & 0\end{array}\right]$.

Proof. Firstly, note that, in account of (20) and (21), for each $z$, the characteristic polynomial of $\mathcal{B}_{n}(z)$ is given by

$$
\nu_{n}(s)=\beta_{n, 2} s^{2}+\beta_{n, 1} s+\beta_{n, 0} I, \quad \beta_{n, 2}=1, \beta_{n, 1}=-n A, \beta_{n, 0}=\operatorname{det}\left(\mathcal{B}_{n}\right) .
$$

Hence, from the Cayley-Hamilton Theorem, $\mathcal{B}_{n}$ satisfies

$$
\mathcal{B}_{n}^{2}-n A \mathcal{B}_{n}+\operatorname{det}\left(\mathcal{B}_{n}\right) I=0_{2 \times 2}, \quad n \geq 1 .
$$

Let us compute $\sum_{k=0}^{2} \beta_{n-1, k}\left(\mathcal{B}_{n}^{k} X-X \mathcal{B}_{n-1}^{k}\right)$. After rearranging, we obtain

$$
\begin{aligned}
\sum_{k=0}^{2} \beta_{n-1, k}\left(\mathcal{B}_{n}^{k} X-X \mathcal{B}_{n-1}^{k}\right)=\left(\mathcal{B}_{n}^{2}-(n-1)\right. & \left.A \mathcal{B}_{n}+\operatorname{det}\left(\mathcal{B}_{n-1}\right)\right) X \\
& -X\left(\mathcal{B}_{n-1}^{2}-(n-1) A \mathcal{B}_{n-1}+\operatorname{det}\left(\mathcal{B}_{n-1}\right)\right) .
\end{aligned}
$$

In account of (28) and (29), the previous equality yields

$$
\sum_{k=0}^{2} \beta_{n-1, k}\left(\mathcal{B}_{n}^{k} X-X \mathcal{B}_{n-1}^{k}\right)=\nu_{n-1}\left(\mathcal{B}_{n}\right) X
$$

Now let us use $\left[28\right.$, Lemma 2.1] $\mathcal{B}_{n}^{k} X-X \mathcal{B}_{n-1}^{k}=\sum_{j=0}^{k-1} \mathcal{B}_{n}^{k-1-j} \mathcal{C}_{n} \mathcal{B}_{n-1}^{j}, \quad k \geq 1$, in (30). After cancelation of common terms, there follows (27). 
Corollary 3.4. Under the conditions of Theorem 3.1, the matrix Sylvester equations (18) are equivalent to

$$
\begin{aligned}
& a_{n}\left(l_{n, 1}(z)+l_{n-1,1}(z)-(n-1) A(z)\right)=\Theta_{n, 1}(z)-z \Theta_{n-1,1}(z), \\
& l_{n, 1}(z)-l_{n-1,1}(z)=A(z)-a_{n} \Theta_{n, 2}(z)+z \bar{a}_{n} \Theta_{n-1,1}(z) .
\end{aligned}
$$

In order to illustrate the results contained in Theorem 3.1 and Corollary 3.4, namely on the obtention of difference equations for the reflection coefficients of $\left\{\phi_{n}\right\}_{n \geq 0}$ through Eqs. (31)-(32), we we shall make use of the results that follow.

Corollary 3.5. Let $\left\{\phi_{n}\right\}_{n \geq 0}$ be a MOPS related to a Carathéodory function $F$ satisfying $z A F^{\prime}=C F+D$ with $\max \{\operatorname{deg}(A), \operatorname{deg}(C)\} \leq 2$. Set $A(z)=\alpha_{2} z^{2}+\alpha_{1} z+\alpha_{0}, C(z)=$ $c_{2} z^{2}+c_{1} z+c_{0}, \phi_{n}(z)=z^{n}+\tau_{n} z^{n-1}+\cdots+k_{n} z+a_{n}$. For all $n \geq 1$, the polynomials $l_{n, 1}, \Theta_{n, j}, j=1,2$ in Eqs. (31)-(32) are

$$
\begin{aligned}
& \Theta_{n, 1}(z)=\theta_{n, 1}^{1} z+\theta_{n, 1}^{0}, \\
& \Theta_{n, 2}(z)=\theta_{n, 2}^{2} z^{2}+\theta_{n, 2}^{1} z, \\
& l_{n, 1}(z)=\left(n \alpha_{2}+\frac{c_{2}}{2}\right) z^{2}+\left(-\alpha_{2} \tau_{n}+n \alpha_{1}+\theta_{n, 1}^{1} \bar{a}_{n}+c_{1} / 2\right) z+n \alpha_{0}-\frac{c_{0}}{2},
\end{aligned}
$$

with

$$
\begin{aligned}
& \theta_{n, 1}^{1}=-\left((n+1) \alpha_{2}+c_{2}\right) a_{n+1}, \quad \theta_{n, 1}^{0}=\left(n \alpha_{0}-c_{0}\right) a_{n}, \\
& \theta_{n, 2}^{2}=-\left(n \alpha_{2}+c_{2}\right) \bar{a}_{n}, \quad \theta_{n, 2}^{1}=\left((n+1) \alpha_{0}-c_{0}\right) \bar{a}_{n+1} .
\end{aligned}
$$

Thus, the following equations hold:

$$
\begin{aligned}
& a_{n}\left[-\alpha_{2}\left(\tau_{n}+\tau_{n-1}\right)+n \alpha_{1}+c_{1}+\theta_{n, 1}^{1} \bar{a}_{n}+\theta_{n-1,1}^{1} \bar{a}_{n-1}\right]-\theta_{n, 1}^{1}=-\theta_{n-1,1}^{0}, \\
& -a_{n} \bar{a}_{n+1}\left((n+1) \alpha_{0}-c_{0}\right)-\theta_{n, 1}^{1} \bar{a}_{n} \\
& \quad=-a_{n-1} \bar{a}_{n}\left(n \alpha_{0}-c_{0}\right)-\theta_{n-1,1}^{1} \bar{a}_{n-1}+\alpha_{0} a_{n-1} \bar{a}_{n}-\alpha_{2} \bar{a}_{n-1} a_{n},
\end{aligned}
$$

Proof. In account of (25), the $\Theta_{n}$ 's are obtained through the expansions (12), (15) and (16), thus we obtain (33)-(35).

Plugging (33)-(35) into (31) and equating the coefficients of $z^{k}, k=0,1,2$, we obtain (36). Also, by plugging (33)-(35) into (32) and equating the coefficients of $z^{k}, k=0,1,2$, we obtain

$$
\begin{aligned}
-a_{n} \bar{a}_{n+1}\left((n+1) \alpha_{0}-c_{0}\right)+\alpha_{2}\left(\tau_{n}-\tau_{n-1}\right)+\theta_{n-1,1}^{1} \bar{a}_{n-1} & -\theta_{n, 1}^{1} \bar{a}_{n} \\
& =-a_{n-1} \bar{a}_{n}\left((n-1) \alpha_{0}-c_{0}\right) .
\end{aligned}
$$

As $\tau_{n}-\tau_{n-1}=\bar{a}_{n-1} a_{n}$ (cf. (13)), from the above equality we obtain (37).

\section{Difference equations for Laguerre-Hahn affine orthogonal polynomials on the unit circle from matrix Sylvester equations}

The main subject of the present section is the deduction of difference equations for the reflection coefficients of orthogonal polynomials related to Carathéodory functions 
satisfying $z A F^{\prime}=C F+D$ with $\max \{\operatorname{deg}(A), \operatorname{deg}(C)\} \leq 2$. Well-known examples of such families of orthogonal polynomials include the so-called generalized Jacobi polynomials $[20$, Sections 3, 4] (see also $[8,15]$ ), as well as some of its perturbations related to the ones studied in $[4,5]$.

\subsection{Generalized Jacobi polynomials on the unit circle: general difference equations and $d P_{V}$}

We start by analysing the so-called generalized Jacobi polynomials on the unit circle $[20$, Section 4], which will be taken as monic,

$$
\phi_{n}(z)=z^{n}+\tau_{n} z^{n-1}+\cdots+k_{n} z+a_{n}, \quad n \geq 0 .
$$

$\left\{\phi_{n}\right\}_{n \geq 0}$ is orthogonal with respect to the weight function

$$
w(z)=k z^{\tilde{\gamma}}\left(z-\eta_{1}\right)^{2 \alpha}\left(z-\eta_{2}\right)^{2 \beta}, \quad \eta_{1}=e^{i \theta_{1}}, \eta_{2}=e^{i \theta_{2}}, k \in \mathbb{C}, \alpha, \beta \in \mathbb{R},
$$

where $\tilde{\gamma}=i \gamma-\alpha-\beta$, being $\gamma$ a real number. $w$ is real on $\mathbb{T}$ and it satisfies $\frac{w^{\prime}}{w}=\frac{C}{z A}$ with

$$
\begin{aligned}
& A(z)=\left(z-\eta_{1}\right)\left(z-\eta_{2}\right) \\
& C(z)=(\alpha+\beta+i \gamma) z^{2}+\left[\eta_{1}(\alpha-\beta-i \gamma)-\eta_{2}(\alpha-\beta+i \gamma)\right] z+\eta_{1} \eta_{2}(i \gamma-\alpha-\beta)
\end{aligned}
$$

The Carathéodory function of $w, F$, satisfies the differential equation

$$
z A F^{\prime}=C F+D
$$

where $A, C$ are the same as in (39)-(40) and $D$ is a polynomial with $\operatorname{deg}(D) \leq 2$. By plugging the data of (39)-(40) in (33)-(35), one obtains Corollary 3.4 with the polynomials $l_{n, 1}$ and $\Theta_{n, j}, j=1,2$, given by

$$
\begin{gathered}
\Theta_{n, 1}(z)=-(n+1+\alpha+\beta+i \gamma) a_{n+1} z+\eta_{1} \eta_{2}(n+\alpha+\beta-i \gamma) a_{n}, \\
\Theta_{n, 2}(z)=-(n+\alpha+\beta+i \gamma) \bar{a}_{n} z^{2}+\eta_{1} \eta_{2}(n+1+\alpha+\beta-i \gamma) \bar{a}_{n+1} z, \\
l_{n, 1}(z)=\left(n+\frac{\alpha+\beta+i \gamma}{2}\right) z^{2}+\ell_{n, 1} z+\eta_{1} \eta_{2} \frac{2 n+\alpha+\beta-i \gamma}{2}, \\
\ell_{n, 1}=-\tau_{n}-\left(\eta_{1}+\eta_{2}\right)\left(n+\frac{i \gamma}{2}\right)+\left(\eta_{1}-\eta_{2}\right)\left(\frac{\alpha-\beta}{2}\right)-(n+1+\alpha+\beta+i \gamma) a_{n+1} \bar{a}_{n} .
\end{gathered}
$$

Proposition 4.1. Let $\left\{\phi_{n}\right\}_{n \geq 0}$ be the MOPS related to the Jacobi weight (38). There holds, for all $n \geq 1$ :

(a) the reflection coefficients satisfy the difference equations

$$
\begin{aligned}
(n+1+\alpha & +\beta+i \gamma) a_{n+1} \bar{a}_{n}-(n-1+\alpha+\beta+i \gamma) a_{n} \bar{a}_{n-1} \\
& =\eta_{1} \eta_{2}\left((n+1+\alpha+\beta-i \gamma) \bar{a}_{n+1} a_{n}-(n-1+\alpha+\beta-i \gamma) \bar{a}_{n} a_{n-1}\right)
\end{aligned}
$$

(b) the coefficients $\tau_{n}$ satisfy the difference equations

$$
(n+1+\alpha+\beta+i \gamma) \tau_{n+1}-\eta_{1} \eta_{2}(n+1+\alpha+\beta-i \gamma) \bar{\tau}_{n+1}=(n+1) \delta_{1},
$$


with

$$
\delta_{1}=(1+\alpha+\beta+i \gamma) a_{1}-\eta_{1} \eta_{2}(1+\alpha+\beta-i \gamma) \bar{a}_{1}
$$

(c) the coefficients $\tau_{n}$ are related to the $a_{n}$ 's through the equation

$$
\begin{aligned}
\tau_{n}= & \frac{(n+1+\alpha+\beta+i \gamma)}{2}\left(\frac{a_{n+1}}{a_{n}}-a_{n+1} \bar{a}_{n}\right)-\frac{(n-1+\alpha+\beta+i \gamma)}{2} a_{n} \bar{a}_{n-1} \\
& +\eta_{1} \eta_{2} \frac{(n-1+\alpha+\beta-i \gamma)}{2} \frac{a_{n-1}}{a_{n}}-\frac{(n+i \gamma)}{2}\left(\eta_{1}+\eta_{2}\right)+\frac{(\alpha-\beta)}{2}\left(\eta_{1}-\eta_{2}\right)
\end{aligned}
$$

In (a)-(c), there holds the initial conditions $a_{0}=1, a_{1}=-\bar{u}_{1} / u_{0}$, where $u_{0}$ and $u_{1}$ are the moments of the weight (38).

Proof. Eq. (44) follows by plugging the data of $A, C$ (cf. (39)-(40)) into (37).

To obtain (45) we sum (44),

$$
\sum_{k=1}^{n}\left(A_{k}-A_{k-1}\right)+\sum_{k=1}^{n} a_{k} \bar{a}_{k-1}=\eta_{1} \eta_{2} \sum_{k=1}^{n}\left(B_{k}-B_{k-1}\right)+\eta_{1} \eta_{2} \sum_{k=1}^{n} \bar{a}_{k} a_{k-1},
$$

where $A_{k}=(k+1+\alpha+\beta+i \gamma) a_{k+1} \bar{a}_{k}, B_{k}=(k+1+\alpha+\beta-i \gamma) \bar{a}_{k+1} a_{k}$, thus obtaining

$$
A_{n}-A_{0}+\tau_{n}=\eta_{1} \eta_{2}\left(B_{n}-B_{0}\right)+\eta_{1} \eta_{2} \bar{\tau}_{n}
$$

In turn, summing (48), after some basic computations, we obtain (45).

Eq. (47) follows by plugging the data of $A, C$ into (36) and using (13).

Proposition 4.2. The reflection coefficients of the MOPS $\left\{\phi_{n}\right\}_{n \geq 0}$ related to the Jacobi weight (38) can be obtained recursively through

$$
\begin{aligned}
(n+1+\alpha+\beta+i \gamma) a_{n+1}=\left(\eta_{1} \eta_{2} \bar{\tau}_{n}\right. & \left.+\tau_{n}+n\left(\eta_{1}+\eta_{2}\right)\right) \frac{a_{n}}{1-\left|a_{n}\right|^{2}} \\
& -\eta_{1} \eta_{2}(n-1+\alpha+\beta-i \gamma) a_{n-1}, \quad n \geq 1,
\end{aligned}
$$

with initial conditions $a_{0}=1, a_{1}=-\bar{u}_{1} / u_{0}$, where $u_{0}$ and $u_{1}$ are the moments of the weight (38).

Proof. Evaluating (31) and (32) at $\eta_{1}$ (respectively $\eta_{2}$ ) and summing the result, we obtain, after some computations, respectively,

$$
\begin{aligned}
& \left((n+1+\alpha+\beta+i \gamma) a_{n+1}+\eta_{1} \eta_{2}(n-1+\alpha+\beta-i \gamma) a_{n-1}\right) \frac{1-\left|a_{n}\right|^{2}}{a_{n}} \\
& =\tau_{n}+\eta_{1} \eta_{2} \bar{\tau}_{n}+\delta_{1}-2\left(\alpha \eta_{1}+(\beta-i \gamma) \eta_{2}\right)+\eta_{2}(n+\alpha+\beta-i \gamma)+\eta_{1}(n+\alpha+\beta+i \gamma), \\
& \left((n+1+\alpha+\beta+i \gamma) a_{n+1}+\eta_{1} \eta_{2}(n-1+\alpha+\beta-i \gamma) a_{n-1}\right) \frac{1-\left|a_{n}\right|^{2}}{a_{n}} \\
& =\tau_{n}+\eta_{1} \eta_{2} \bar{\tau}_{n}+\delta_{1}-2\left(\beta \eta_{2}+(\alpha-i \gamma) \eta_{1}\right)+\eta_{1}(n+\alpha+\beta-i \gamma)+\eta_{2}(n+\alpha+\beta+i \gamma),
\end{aligned}
$$


with $\delta_{1}$ given in (46). Summing the above equations and dividing the result by 2 yields

$$
\begin{aligned}
\left((n+1+\alpha+\beta+i \gamma) a_{n+1}+\eta_{1} \eta_{2}(n-1+\alpha+\beta-i \gamma) a_{n-1}\right) \frac{1-\left|a_{n}\right|^{2}}{a_{n}} & \\
& =\tau_{n}+\eta_{1} \eta_{2} \bar{\tau}_{n}+\lambda+n\left(\eta_{1}+\eta_{2}\right),
\end{aligned}
$$

where

$$
\lambda=\delta_{1}+(\alpha-\beta)\left(\eta_{2}-\eta_{1}\right)+i \gamma\left(\eta_{1}+\eta_{2}\right)
$$

Recall that, in account of (5), $\delta_{1}$ is given in terms of the moments $u_{0}$ and $u_{1}$ of $w$, $\delta_{1}=-(1+\alpha+\beta+i \gamma) \frac{\bar{u}_{1}}{u_{0}}+\eta_{1} \eta_{2}(1+\alpha+\beta-i \gamma) \frac{u_{1}}{u_{0}}$. Thus, $\lambda=0$ and (49) follows.

Remark 4.3. Eq. (49) is Eq. (24) in [20].

The discrete Painlevé $V\left(d P_{V}\right)$ system is deduced in the theorem that follows.

Theorem 4.4. The reflection coefficients of the MOPS $\left\{\phi_{n}\right\}_{n \geq 0}$ related to the Jacobi weight (38) satisfy the system of discrete Painlevé equations, for all $n \geq 1$,

$$
\begin{aligned}
F_{n}+F_{n-1}= & 2 \alpha+\frac{n-1+\alpha+\beta-i \gamma}{G_{n}-1}+\frac{\eta_{1}(n+\alpha+\beta+i \gamma)}{G_{n}-\eta_{1} / \eta_{2}} \\
& \frac{\left(F_{n}+n\right)\left(F_{n}+n+2 \beta\right)}{F_{n}\left(F_{n}-2 \alpha\right)}=\frac{\eta_{2}}{\eta_{1}} G_{n+1} G_{n}
\end{aligned}
$$

with

$$
\begin{aligned}
F_{n} & =\frac{1}{\eta_{2}-\eta_{1}}\left(-\tau_{n}-n \eta_{2}+\frac{(n+1+\alpha+\beta+i \gamma) a_{n+1}}{a_{n}}\left(1-\left|a_{n}\right|^{2}\right)\right), \\
G_{n} & =\frac{\eta_{1}(n-1+\alpha+\beta-i \gamma)-(n+\alpha+\beta+i \gamma) a_{n} / a_{n-1}}{\eta_{2}(n-1+\alpha+\beta-i \gamma)-(n+\alpha+\beta+i \gamma) a_{n} / a_{n-1}},
\end{aligned}
$$

subject to the initial conditions $F_{0}=0, G_{1}=\frac{\eta_{1}(\alpha+\beta-i \gamma)-(1+\alpha+\beta+i \gamma) a_{1}}{\eta_{2}(\alpha+\beta-i \gamma)-(1+\alpha+\beta+i \gamma) a_{1}}$.

Proof. Let us write, for $j=1,2$, in account of (41)-(43),

$$
\begin{aligned}
& \Theta_{n, 1}\left(\eta_{j}\right)=\eta_{j}\left(-N_{n+1} a_{n+1}+\bar{N}_{n} \eta_{\pi_{j}} a_{n}\right), \\
& \Theta_{n, 2}\left(\eta_{j}\right)=\eta_{j}^{2}\left(-N_{n} \bar{a}_{n}+\bar{N}_{n+1} \eta_{\pi_{j}} \bar{a}_{n+1}\right), \\
& l_{n, 1}\left(\eta_{j}\right)=\eta_{j}\left(\rho_{j}-\tau_{n}-N_{n+1} \bar{a}_{n} a_{n+1}\right),
\end{aligned}
$$

where

$$
\begin{gathered}
\rho_{1}=\alpha \eta_{1}+(\beta-i \gamma) \eta_{2}, \rho_{2}=\beta \eta_{2}+(\alpha-i \gamma) \eta_{1}, N_{n}=n+\alpha+\beta+i \gamma \\
\pi_{j}=2 \text { if } j=1, \pi_{j}=1 \text { if } j=2 .
\end{gathered}
$$

Firstly we deduce (51). This equation follows from the use of (31) evaluated at $\eta_{j}$. Let us detail. 
By evaluating (31) at $\eta_{1}$ we obtain, in account of (56)-(57),

$$
F_{n}^{1}+F_{n-1}^{1}=-2 \rho_{1}+\eta_{2} \bar{N}_{n}+\eta_{1} N_{n}-\eta_{1} \eta_{2} \bar{N}_{n-1} \frac{a_{n-1}}{a_{n}}+N_{n} \frac{a_{n}}{a_{n-1}}
$$

with $F_{n}^{1}=-\tau_{n}+N_{n+1} \frac{a_{n+1}}{a_{n}}\left(1-\left|a_{n}\right|^{2}\right)$. Therefore, we get

$$
\hat{F}_{n}+\hat{F}_{n-1}=2 \alpha\left(\eta_{2}-\eta_{1}\right)-\eta_{2} \bar{N}_{n-1}+\eta_{1} N_{n}-\eta_{1} \eta_{2} \bar{N}_{n-1} \frac{a_{n-1}}{a_{n}}+N_{n} \frac{a_{n}}{a_{n-1}}
$$

with $\hat{F}_{n}=F_{n}^{1}-\eta_{2} \bar{N}_{n}+(\alpha+\beta-i \gamma) \eta_{2}$.

As $-\eta_{2} \bar{N}_{n}+(\alpha+\beta-i \gamma) \eta_{2}=-n \eta_{2}$, (58) is given by

$$
\tilde{F}_{n}+\tilde{F}_{n-1}=2 \alpha\left(\eta_{2}-\eta_{1}\right)-\left(\eta_{2} \bar{N}_{n-1}-N_{n} \frac{a_{n}}{a_{n-1}}\right)-\eta_{1}\left(\frac{\eta_{2} \bar{N}_{n-1}-N_{n} a_{n} / a_{n-1}}{a_{n} / a_{n-1}}\right)
$$

with $\tilde{F}_{n}=F_{n}^{1}-n \eta_{2}$, equivalently,

$$
\tilde{F}_{n}+\tilde{F}_{n-1}=2 \alpha\left(\eta_{2}-\eta_{1}\right)+\frac{\left(\eta_{2}-\eta_{1}\right) \bar{N}_{n-1}}{\frac{-\left(\eta_{2}-\eta_{1}\right) \bar{N}_{n-1}}{\eta_{2} \bar{N}_{n-1}-N_{n} a_{n} / a_{n-1}}}+\frac{\eta_{1}\left(\eta_{2}-\eta_{1}\right) N_{n}}{\frac{-\left(\eta_{2}-\eta_{1}\right) N_{n} a_{n} / a_{n-1}}{\eta_{2} \bar{N}_{n-1}-N_{n} a_{n} / a_{n-1}}}
$$

Therefore, we obtain

$$
\frac{1}{\eta_{2}-\eta_{1}}\left(\tilde{F}_{n}+\tilde{F}_{n-1}\right)=2 \alpha+\frac{\bar{N}_{n-1}}{G_{n}-1}+\frac{\eta_{1} N_{n}}{G_{n}-\eta_{1} / \eta_{2}}
$$

with $G_{n}=\frac{\eta_{1} \bar{N}_{n-1}-N_{n} a_{n} / a_{n-1}}{\eta_{2} \bar{N}_{n-1}-N_{n} a_{n} / a_{n-1}}$. Hence, we have (51) with $F_{n}$ and $G_{n}$ given by (53) and (54), respectively.

Let us now deduce (52). This equation follows from the use of (21) evaluated at $\eta_{j}$. Let us detail.

By evaluating (21) at $\eta_{1}$ we obtain

$$
\frac{l_{n, 1}^{2}\left(\eta_{1}\right)}{\eta_{1}^{2}}-\frac{b_{1}}{\eta_{1}^{2}}=-\frac{\Theta_{n, 1}\left(\eta_{1}\right) \Theta_{n, 2}\left(\eta_{1}\right)}{\eta_{1}^{2}}, \quad b_{1}=-\operatorname{det}\left(\mathcal{B}_{1}\right)\left(\eta_{1}\right) .
$$

Recall the relations (cf. (57))

$$
\frac{l_{n, 1}\left(\eta_{1}\right)}{\eta_{1}}=\left(\eta_{2}-\eta_{1}\right) F_{n}+\rho_{1}+n \eta_{2}-N_{n+1} \frac{a_{n+1}}{a_{n}}, \rho_{1}+n \eta_{2}=\eta_{2} \bar{N}_{n}-\alpha\left(\eta_{2}-\eta_{1}\right),
$$

as well as, in account of (22) and (40), $b_{1}=\alpha^{2} \eta_{1}^{2}\left(\eta_{2}-\eta_{1}\right)^{2}$, which yield the left hand side of (60) given by

$$
\begin{aligned}
& \left(\eta_{2}-\eta_{1}\right)^{2}\left(\left(F_{n}-\alpha\right)^{2}-\alpha^{2}\right) \\
& \quad+\left(\eta_{2} \bar{N}_{n}-N_{n+1} \frac{a_{n+1}}{a_{n}}\right)\left(\eta_{2} \bar{N}_{n}-N_{n+1} \frac{a_{n+1}}{a_{n}}+2\left(\eta_{2}-\eta_{1}\right)\left(F_{n}-\alpha\right)\right) .
\end{aligned}
$$


Therefore, in account of (55)-(56), Eq. (60) yields

$$
\begin{aligned}
& \left(\eta_{2}-\eta_{1}\right)^{2} F_{n}\left(F_{n}-2 \alpha\right) \\
& =\left(-\eta_{2} \bar{N}_{n}+N_{n+1} \frac{a_{n+1}}{a_{n}}\right)\left(\eta_{2} \bar{N}_{n}-N_{n+1} \frac{a_{n+1}}{a_{n}}+2\left(\eta_{2}-\eta_{1}\right)\left(F_{n}-\alpha\right)\right) \\
& \quad+\left(N_{n+1} \frac{a_{n+1}}{a_{n}}-\eta_{2} \bar{N}_{n}\right)\left(-\eta_{1} N_{n}\left|a_{n}\right|^{2}+\eta_{1} \eta_{2} \bar{N}_{n+1} a_{n} \bar{a}_{n+1}\right) .
\end{aligned}
$$

Using $F_{n}$ given by (53) in the right-hand side of the above equation we obtain

$$
\left(\eta_{2}-\eta_{1}\right)^{2} F_{n}\left(F_{n}-2 \alpha\right)=\eta_{1} \frac{a_{n-1}}{a_{n}}\left(1-\left|a_{n}\right|^{2}\right) \times \text { denominator of } G_{n+1} G_{n} .
$$

Proceeding by an analogous manner, starting by evaluating (21) at $\eta_{2}$ we obtain

$$
\frac{l_{n, 1}^{2}\left(\eta_{2}\right)}{\eta_{2}^{2}}-\frac{b_{2}}{\eta_{2}^{2}}=-\frac{\Theta_{n, 1}\left(\eta_{2}\right) \Theta_{n, 2}\left(\eta_{2}\right)}{\eta_{2}^{2}}, \quad b_{2}=-\operatorname{det}\left(\mathcal{B}_{1}\right)\left(\eta_{2}\right) .
$$

Recall the relations (cf. (57))

$$
\frac{l_{n, 1}\left(\eta_{2}\right)}{\eta_{2}}=\left(\eta_{2}-\eta_{1}\right) F_{n}+\rho_{2}+n \eta_{2}-N_{n+1} \frac{a_{n+1}}{a_{n}},
$$

which yield the left hand side of (62) given by

$$
\left(\left(\eta_{2}-\eta_{1}\right)\left(F_{n}+n\right)-N_{n+1} \frac{a_{n+1}}{a_{n}}+\rho_{2}+n \eta_{1}\right)^{2}-\frac{b_{2}}{\eta_{2}^{2}}
$$

equivalently, in account of $\rho_{2}+n \eta_{1}=\eta_{1} \bar{N}_{n}+\beta\left(\eta_{2}-\eta_{1}\right)$ and $b_{2}=\beta^{2} \eta_{2}^{2}\left(\eta_{2}-\eta_{1}\right)^{2}$,

$$
\begin{aligned}
\left(\eta_{2}-\eta_{1}\right)^{2} & \left(\left(F_{n}+n+\beta\right)^{2}-\beta^{2}\right) \\
& +\left(\eta_{1} \bar{N}_{n}-N_{n+1} \frac{a_{n+1}}{a_{n}}\right)\left(\eta_{1} \bar{N}_{n}-N_{n+1} \frac{a_{n+1}}{a_{n}}+2\left(\eta_{2}-\eta_{1}\right)\left(F_{n}+n+\beta\right)\right) .
\end{aligned}
$$

Therefore, in account of (55)-(56), Eq. (62) yields

$$
\begin{aligned}
& \left(\eta_{2}-\eta_{1}\right)^{2}\left(F_{n}+n\right)\left(F_{n}+n+2 \beta\right) \\
& =\left(-\eta_{1} \bar{N}_{n}+N_{n+1}\right. \\
& \left.\quad \frac{a_{n+1}}{a_{n}}\right)\left(\eta_{1} \bar{N}_{n}-N_{n+1} \frac{a_{n+1}}{a_{n}}+2\left(\eta_{2}-\eta_{1}\right)\left(F_{n}+n+\beta\right)\right) \\
& \quad+\left(N_{n+1} \frac{a_{n+1}}{a_{n}}-\eta_{1} \bar{N}_{n}\right)\left(-\eta_{2} N_{n}\left|a_{n}\right|^{2}+\eta_{1} \eta_{2} \bar{N}_{n+1} a_{n} \bar{a}_{n+1}\right) .
\end{aligned}
$$

Using $F_{n}$ given by (53) in the right-hand side of the above equation we obtain

$$
\left(\eta_{2}-\eta_{1}\right)^{2}\left(F_{n}+n\right)\left(F_{n}+n+2 \beta\right)=\eta_{2} \frac{a_{n-1}}{a_{n}}\left(1-\left|a_{n}\right|^{2}\right) \times \text { numerator of } G_{n+1} G_{n} .
$$

Taking the ratio between (61) and (63) we get (52). 
Remark 4.5. If we take $\left\{\eta_{1}, \eta_{2}\right\}=\{-1,-1 / t\}$ in (38), then the difference equations contained in Propositions 4.1 and 4.2 as well as in Theorem 4.4 agree with the ones given in [15], in Lemmas 3.2, 3.3, 3.4, Corollary 3.1, and Proposition 3.4.

\subsection{Further results: difference equations for perturbations of generalized Jacobi polynomials}

Let $w$ be the Jacobi weight $(38)$ and $\left(u_{n}\right)_{n \in \mathbb{Z}}$ its moments. For a fixed non-negative integer $j$, we define the weight $w^{[j]}$, with moments $\left(u_{n}^{[j]}\right)_{n \in \mathbb{Z}}$, through the perturbations

$$
\begin{aligned}
& u_{n}^{[0]}= \begin{cases}u_{n}, & n \neq 0, \\
u_{0}+m, & n=0\end{cases} \\
& u_{n}^{[j]}= \begin{cases}u_{n}, & n \notin\{-j, j\}, \quad j \geq 1, \\
u_{n}+m, & n=j, \\
u_{n}+\bar{m}, & n=-j\end{cases}
\end{aligned}
$$

where, in (64), $m \in \mathbb{R}$ and, in (65), $m \in \mathbb{C}$.

Remark 4.6. The above transformations are connected to perturbations of Hermitian matrices [5]. In the language of Hermitian linear functionals, the functional related to $w^{[j]}$ is a sum perturbation of the functional $u$ related to $w$, given as

$$
u^{[j]}= \begin{cases}u+m \mathcal{L}, & j=0, \\ u+2 \Re e\left(m z^{j}\right) \mathcal{L}, & j \geq 1\end{cases}
$$

with $\mathcal{L}$ the normalized Lebesgue functional. Necessary and sufficient conditions for the regularity of linear functionals $u^{[j]}$ of the above type were given in [5, Propositions 1 and 7 .

The Carathéodory function of $w^{[j]}, F^{[j]}$, is given in terms of the Carathéodory function of $w$ as

$$
F^{[j]}(z)= \begin{cases}F(z)+m, & j=0 \\ F(z)+2 m z^{j}, & j \geq 1 .\end{cases}
$$

As $F$ satisfies $z A F^{\prime}=C F+D$, then we have

$$
z A\left(F^{[j]}\right)^{\prime}=C F^{[j]}+D+ \begin{cases}-m C, & j=0 \\ 2 m z^{j}(j A-C), & j \geq 1\end{cases}
$$

Thus, $F^{[j]}$ is Laguerre-Hahn affine such that the condition $\max \{\operatorname{deg}(A), \operatorname{deg}(C)\} \leq 2$ is preserved.

In the sequel we assume the regularity of $u^{[j]}$ defined by (66), that is, we assume the regularity of the weight $w^{[j]}$. We denote the MOPS related to $w^{[j]}$ by $\left\{\phi_{n}^{[j]}\right\}_{n \geq 0}$ and we write

$$
\phi_{n}^{[j]}(z)=z^{n}+\tau_{j, n} z^{n-1}+\cdots+k_{j, n} z+a_{j, n}, \quad n \geq 0 .
$$


In account of (5), there holds $a_{j, 0}=1, j \geq 0$,

$$
a_{0, n}=\frac{(-1)^{n} \operatorname{det}\left(\widehat{\mathbf{T}}_{n}+m \mathbf{Z}_{n}^{t}\right)}{\operatorname{det}\left(\mathbf{T}_{n-1}+m I_{n}\right)}, \quad n \geq 1
$$

and, for all $j \geq 1, n \geq 1$,

$$
a_{j, n}= \begin{cases}\frac{a_{n},}{(-1)^{n} \operatorname{det}\left(\widehat{\mathbf{T}}_{n}+\bar{m} \mathbf{Z}_{n}^{j-1}\right)} & n \leq j-1, \\ \Delta_{n-1} & n=j, \\ \frac{(-1)^{n} \operatorname{det}\left(\widehat{\mathbf{T}}_{n}+\bar{m} \mathbf{Z}_{n}^{j-1}\right)}{\operatorname{det}\left(\mathbf{T}_{n-1}+\bar{m} \mathbf{Z}^{j}+m\left(\mathbf{Z}_{n}^{t}\right)^{j}\right)}, & n=j+1, \\ \frac{(-1)^{n} \operatorname{det}\left(\widehat{\mathbf{T}}_{n}+\bar{m} \mathbf{Z}_{n}^{j-1}+m\left(\mathbf{Z}_{n}^{t}\right)^{j+1}\right)}{\operatorname{det}\left(\mathbf{T}_{n-1}+\bar{m} \mathbf{Z}_{n}^{j}+m\left(\mathbf{Z}_{n}^{t}\right)^{j}\right)}, & n \geq j+2,\end{cases}
$$

where $\widehat{\mathbf{T}}_{n}=\left[\begin{array}{cccc}\bar{u}_{1} & \bar{u}_{2} & \ldots & \bar{u}_{n} \\ u_{0} & \bar{u}_{1} & \ldots & \bar{u}_{n-1} \\ \vdots & & \ddots & \vdots \\ u_{n-2} & \ldots & u_{0} & \bar{u}_{1}\end{array}\right], \mathbf{Z}_{n}$ is the shift matrix of order $n$ with ones on the first upper-diagonal and zeroes on the remaining entries, $t$ denotes the transpose, and $I_{n}$ is the identity matrix of order $n$.

Theorem 4.7. Under the notations previously defined, for each non-negative integer $j$, the following statements hold:

(a) the reflection coefficients of the MOPS $\left\{\phi_{n}^{[j]}\right\}_{n \geq 0}$ satisfy Eqs. (44), (45), (47) where, for all $n \geq j+1$, the initial conditions $a_{j, j}, a_{j, j+1}$ given by (68)-(69) take place;

(b) for all $n \geq j+1$, the reflection coefficients of the MOPS $\left\{\phi_{n}^{[j]}\right\}_{n \geq 0}$ can be obtained recursively through

$$
\begin{aligned}
(n+1+\alpha+\beta+i \gamma) a_{j, n+1}=\left(\eta_{1} \eta_{2} \bar{\tau}_{j, n}+\tau_{j, n}\right. & \left.+n\left(\eta_{1}+\eta_{2}\right)+\lambda_{j}\right) \frac{a_{j, n}}{1-\left|a_{j, n}\right|^{2}} \\
& -\eta_{1} \eta_{2}(n-1+\alpha+\beta-i \gamma) a_{j, n-1},
\end{aligned}
$$

with $\lambda_{j}=\delta_{j, 1}+(\alpha-\beta)\left(\eta_{2}-\eta_{1}\right)+i \gamma\left(\eta_{1}+\eta_{2}\right)$,

$$
\delta_{j, 1}= \begin{cases}-(1+\alpha+\beta+i \gamma) \frac{\bar{u}_{1}}{u_{0}+m}+\eta_{1} \eta_{2}(1+\alpha+\beta-i \gamma) \frac{u_{1}}{u_{0}+m}, & j=0, \\ -(1+\alpha+\beta+i \gamma) \frac{\bar{u}_{1}}{u_{0}}+\eta_{1} \eta_{2}(1+\alpha+\beta-i \gamma) \frac{u_{1}}{u_{0}}, & j \geq 1,\end{cases}
$$

with initial conditions $a_{j, j}, a_{j, j+1}$ given by (68)-(69);

(c) the reflection coefficients of the MOPS $\left\{\phi_{n}^{[j]}\right\}_{n \geq 0}$ satisfy the system of discrete Painlevé equations (51)-(52), where, for all $n \geq j+1$, the initial conditions are defined in terms of $a_{j, j}, a_{j, j+1}$ given by (68)-(69).

Proof. Recall that the difference equations deduced in Propositions 4.1 and 4.2 and Theorem 4.4 follow from the difference relations given in Corollary 3.4, and these are based 
on the polynomials $l_{n}$ and $\Theta_{n}$, which depend on the coefficients $A, C$ of the differential equation $z A F^{\prime}=C F+D$. As $F^{[j]}$ satisfies (67) with the same $A, C$, then all the calculus done before remain valid. Hence, the assertions follow. Note that (70) follows from (50).

\section{Acknowledgements}

This manuscript was substantially improved thanks to the suggestions and comments of an anonymous referee. The author is deeply indebt to her/him.

This work was partially supported by the Centre for Mathematics of the University of Coimbra - UID/MAT/00324/2013, funded by the Portuguese Government through FCT/MEC and co-funded by the European Regional Development Fund through the Partnership Agreement PT2020.

\section{References}

[1] Cachafeiro A, Pérez C. A study of the Laguerre-Hahn affine functionals on the unit circle. J Comput Anal Appl. 2004;6:107-123.

[2] Tasis C. Propiedades diferenciales de los polinomios ortogonales relativos a la circunferencia unidad [doctoral dissertation]. Cantabria (Spain): University of Cantabria; 1989.

[3] Branquinho A, Rebocho MN. Distributional equation for Laguerre-Hahn functionals on the unit circle. J Comput Appl Math. 2009;233:634-642.

[4] Cachafeiro A, Marcellán F, Pérez C. Lebesgue perturbation of a quasi-definite Hermitian functional. The positive-definite case. Linear Algebra Appl. 2003;369:235-250.

[5] Castillo K, Garza L, Marcellán F. Perturbations on the subdiagonals of Toeplitz matrices. Linear Algebra Appl. 2001;434:1563-1579.

[6] Witte NS. Bi-orthogonal systems on the unit circle, regular semi-classical weights and integrable systems - II. J Approx Theory. 2009;161:565-616.

[7] Forrester PJ, Ormerod CM. Differential equations for deformed Laguerre polynomials. J Approx Theory. 2010;162:653-677.

[8] Forrester PJ, Witte NS. Bi-orthogonal Polynomials on the Unit Circle, Regular SemiClassical Weights and Integrable Systems. Constr Approx. 2006;24:201-237.

[9] Ismail MEH, Witte NS. Discriminants and functional equations for polynomials orthogonal on the unit circle. J Approx Theory. 2001;110:200-228.

[10] Magnus AP. Painlevé-type differential equations for the recurrence coefficients of semiclassical orthogonal polynomials. J Comput Appl Math. 1995;57:215-237.

[11] Spicer PE, Nijhoff WF. Semi-classical Laguerre polynomials and a third-order discrete integrable equation. J Phys A: Math Theor. 2009;42:454019 (9pp).

[12] Bleher P, Its AR. Semiclassical asymptotics of orthogonal plynomials, Riemann-Hilbert problem, and universality in the matrix model. Ann of Math. 1999;150:185-266.

[13] Chen Y, Ismail MEH. Jacobi polynomials from compatibility conditions. Proc Amer Math Soc. 2004;133:465-472.

[14] Chen Y, Its AR. Painlevé III and a singular linear statistics in Hermitian random matrix ensembles, I. J Approx Theory. 2010;162:270-297.

[15] Forrester PJ, Witte NS. Discrete Painlevé equations for a class of $\mathrm{P}_{V I} \tau$-functions given as $U(N)$ averages. Nonlinearity. 2005;18:2061-88.

[16] Grammaticos B, Ramani A. Discrete Painlevé equations: A review. In: Grammaticos B, Kosmann-Schwarzbach Y, Tamizhmani T, editors. Discrete Integrable Systems. Lect Notes Phys. Vol. 644. Berlin Heidelberg: Springer; 2004. p. 245-321.

[17] Van Assche W. Discrete Painlevé equations for recurrence coefficients of orthogonal polynomials. In: Elaydi S et al., editors. Difference Equations, Special Functions and Orthogonal Polynomials. World Scientific; 2007. p. 687-725. 
[18] Branquinho A, Rebocho MN. Difference and differential equations for deformed LaguerreHahn orthogonal polynomials on the unit circle. J Phys A: Math Theor. 2011;44:465204 (12 pp).

[19] Branquinho A, Rebocho MN. Matrix Sylvester equations in the theory of orthogonal polynomials on the unit circle. Bull Belg Math Soc. 2010;17:355-376.

[20] Magnus AP. MAPA3072A Special topics in approximation theory 1999-2000: Semi-classical orthogonal polynomials on the unit circle. 2000. Available from: http://www.math.ucl.ac.be/ magnus/.

[21] Szegö G. Orthogonal Polynomials. 4th ed. Providence Rhode Island: Amer Math Soc Colloq Publ.; 1975.

[22] Simon B. Orthogonal Polynomials on the Unit Circle - Part 1. 1st ed. Providence Rhode Island: Amer Math Soc Colloq Publ.; 2005.

[23] Golinskii L, Nevai P. Szegő difference equations, transfer matrices and orthogonal polynomials on the unit circle. Commun Math Phys. 2001;223:223-259.

[24] Erdélyi T, Geronimo JS, Nevai P, Zhang J. A simple proof of Favard's Theorem on the unit circle. Atti Sem Mat Fis Univ Modena. 1991;29:41-46.

[25] Peherstorfer F. A special class of polynomials orthogonal on the unit circle including the associated polynomials. Constr Approx 1996;12:161-185.

[26] Peherstorfer F, Steinbauer R. Characterization of orthogonal polynomials with respect to a functional. J Comput Appl Math. 1995;65:339-355.

[27] Abou-Kandil H, Freiling G, Ionescu V, Jank G. Matrix Riccati Equations in Control and Systems Theory. 1st ed. Basel: Birhäuser; 2003.

[28] Hu Q, Cheng D. The polynomial solution to the Sylvester matrix equation. Appl Math Lett. $2006 ; 19: 859-864$. 